\title{
A NOTE ON THE DUALITY OF LOCALLY COMPACT GROUPS
}

\author{
by J. W. BAKER
}

(Received 14 April, 1967)

Let $H$ be a group of characters on an (algebraic) abelian group $G$. In a natural way, we may regard $G$ as a group of characters on $H$. In this way, we obtain a duality between the two groups $G$ and $H$. One may pose several problems about this duality. Firstly, one may ask whether there exists a group topology on $G$ for which $H$ is precisely the set of continuous characters. This question has been answered in the affirmative in [1]. We shall say that such a topology is compatible with the duality between $G$ and $H$. Next, one may ask whether there exists a locally compact group topology on $G$ which is compatible with a given duality and, if so, whether there is more than one such topology. It is this second question (previously considered by other authors, to whom we shall refer below) which we shall consider here.

Henceforth, we shall consider a pair of abelian groups $G$ and $H$ in duality, in the above sense; we shall assume that the duality is separating, that is that, if $x$ is in $G, x \neq e$, then there exists $\chi_{x}$ in $H$ for which $\chi_{x}(x) \neq 1$. In [1], Varopoulos has initiated a study of the duality between abelian groups in such a way as to parallel the duality theory of locally convex spaces. Let $w(G, H)$ denote the weakest topology on $G$ for which every character in $H$ is continuous. This topology is characterised in [1, part 2]. It is clearly a Hausdorff group topology. What is less obvious is that this topology is compatible with the duality between $G$ and $H$ (see [1, Corollary to Proposition 1]).

The key to our search for a locally compact group topology compatible with the duality is provided by the following theorem, due to Glicksberg [2, Theorem 1.2].

TheOREM 1. Let $G$ be a Hausdorff, locally compact, topological abelian group, with charactergroup $H$. A subset $K$ of $G$ is compact if and only if $K$ is compact in the topology $w(G, H)$.

By using the Pontrjagin duality theorem, one can establish that (in this case) the topology of uniform convergence on the $w(G, H)$-compact subsets of $G$ is a locally compact topology on $H$ which is compatible with the duality between $H$ and $G$.

If $G$ and $H$ are groups in duality, we shall denote by $c(G, H)$ the topology (on $G$ ) of uniform convergence on the $w(H, G)$-compact subsets of $H$. In [1], Varopoulos makes use of a topology $\tau(G, H)$. This topology is weaker than $c(G, H)$, but is strong in a certain sense. For our purposes, we shall use a topology which we shall denote by $\pi(G, H)$. This topology has similar properties to $\tau$. It is defined in a similar way to the "almost weak" topology which is studied in the theory of locally convex topological vector spaces. We say that a subset $C$ of $G$ is $\pi(G, H)$-closed if and only if $C \cap K$ is $w(G, H)$-closed for every $w(G, H)$-compact subset $K$ of $G$. It is simple to verify that $\pi$ is the strongest topology which has the same compact subsets as $w(G, H)$. Alternatively, $\pi$ is the strongest topology which agrees with $w$ on the $w(G, H)$ compact sets. This topology is Hausdorf, and makes $G$ into a semi-topological group (see Husain [4] and [5]). We now give an example to show that $G$ is not a topological group, that is, that the binary operation is not jointly continuous, in general.

$\dagger$ Let $E$ denote the real vector space consisting of all real sequences with finite support. † I am grateful to the referee for his help in suggesting the existence of this example. 
Denote by $E^{*}$ the set of all linear functionals on $E$. Consider the topology $\sigma\left(E, E^{*}\right)$ on $E$. If $K$ is a compact subset of $E$, then every linear functional on $K$ is bounded; so $K$ must be contained in a finite dimensional subspace of $E$. On such subspaces, the topology of $E$ is the usual Euclidean topology. Hence the compact subsets of $E$ are precisely the bounded, closed subsets of finite dimensional subspaces of $E$.

Let us denote by $F$ the set of all functionals $\chi=\exp (i f)$, where $f$ is an element of $E^{*}$. Consider the topology $w(E, F)$ on $E$. This is weaker than $\sigma\left(E, E^{*}\right)$. It is again possible to see that a $w(E, F)$-compact set must be contained in a finite dimensional subspace of $E$. If $\left\{x_{n}\right\}$ is a countably infinite, linearly independent subset of $E$, there exists, for each natural number $n$, a character $\chi_{n}$ in $F$ for which $\chi_{n}\left(x_{n}\right)=-1$, and $\chi_{n}\left(x_{k}\right)=1$ if $k \neq n$. Hence the set $\left\{x_{n}\right\}$ is discrete in $E$. Thus it is clear that the set $\left\{x_{n}\right\}$ cannot be relatively compact in $E(w(E, F))$. Hence every compact subset of $E$ must be finite dimensional. Since $R^{n}$ (as a locally compact group) is self-dual, Theorem 1 shows that the $w(E, F)$-compact sets are again the bounded, closed subsets of finite dimensional subsets of $E$.

Now look at the topology $\pi(E, F)$. This is the strongest topology on $E$ which has the same compact subsets as the finite dimensional subsets of $E$, in the Euclidean topology. If $E_{0}$ is a finite dimensional subspace of $E$, then in view of Corollary 2 to Lemma 2 (see below) the topology of $E_{0}$ is precisely the Euclidean topology. Hence each neighbourhood of 0 in $\pi(E, F)$ is absorbent. Consider the set $A=\bigcup_{n=1}^{\infty} A_{n}$ where $A_{n}$ is the set of all sequences $\left\{x_{n}\right\}$ in $E$ for which $\left|x_{k}\right| \leqq n^{-1}$ for $1 \leqq k \leqq n$ and $x_{k}=0$ otherwise. Then $A$ is a neighbourhood of 0 in $\pi(E, F)$, but there does not exist a subset $B$ of $E$ which is absorbent and for which $B+B \subseteq A$. Thus, in this case, the topology $\pi(G, H)$ is not a group topology.

Luckily, we have the following lemma.

LEMmA 1. If the topology $\pi(G, H)$ is locally compact, then it is a group topology.

Proof. It is clear that the topology is invariant under translation, and also under inversion. Let $U$ be a compact neighbourhood of $e$ for $\pi$. Then the set $U+U$ is also compact, since $w$ is a group topology. Since $w$ and $\pi$ agree on compact sets, $U=(U+U) \cap V$, where $V$ is a $w(G, H)$-neighbourhood of $e$. Choose a $w$-neighbourhood $V_{1}$ of $e$ for which $V_{1}+V_{1} \subset V$. Then $V_{1}$ is a $\pi$-neighbourhood of $e$, so that $U \cap V_{1}$ is a $\pi$-neighbourhood of $e$. Also $\left(U \cap V_{1}\right)+\left(U \cap V_{1}\right) \subset(U+U) \cap V=U$. Hence the lemma is established.

LEMMA 2. Let $(X, \rho)$ be a topological space which is either $(a)$ metrisable or $(b)$ locally compact. Let $\pi$ be a topology on $X$ such that $\pi \cap \rho$ is Hausdorff and every $\rho$-compact set is $\pi$-compact. Then $\rho \supseteq \pi$.

Proof. Suppose that $\rho$ is metrisable. Let $\left\{x_{n}\right\} \rightarrow x(\rho)$. We have to show that $\left\{x_{n}\right\} \rightarrow x(\pi)$. Suppose that the sequence does not converge to $x$ in $\pi$. Then there exists a subsequence $\left\{x_{n_{k}}\right\}$ which lies entirely outside some neighbourhood $U$ of $x$. The set $\{x\} \cup\left\{x_{n_{k}}: k=1,2, \ldots\right\}$ is $\rho$-compact, and so is $\pi$-compact. Let $y$ be a cluster point of $\left\{x_{n_{k}}\right\}$ in $(X, \pi)$. Since $\pi \cap \rho$ is Hausdorff, we must have $y=x$. Since $\left\{x_{n_{k}}\right\} \cap U$ is empty, we have a contradiction, and 
part $(a)$ of the lemma is established. The proof of part $(b)$ of the lemma is similar. It is sufficient to replace $\left\{x_{n}\right\}$ by a net $\left\{x_{\alpha}\right\} \rightarrow x(\rho)$, which may be assumed to be relatively compact. We remark that part $(b)$ is essentially the same as the lemma on page 475 of [1].

This lemma has several immediate corollaries.

Corollary 1. (cf. Corollary 2 on page 484 of [1]) Let $(G, \rho)$ be a metrisable topological abelian group whose character group $H$ separates points of $G$; then $\rho \supseteq \pi(G, H)$.

For the proof of this corollary it is sufficient to observe that $w(G, H) \subseteq \rho \cap \pi(G, H)$ is Hausdorff, and that, if $K$ is $\rho$-compact, then $K$ is $w$-compact and so $\pi$-compact. This argument is also valid if $\rho$ is locally compact. However, in view of Theorem 1 , if $\rho$ is locally compact, then $\pi \supseteq \rho$. Therefore $\pi=\rho$.

COROLLARY 2. Let $G$ be a locally compact abelian group with character group $H$. Then the topology of $G$ is precisely $\pi(G, H)$.

COROLLARY 3. If $G$ is an abelian group and $H$ is a set of characters on $G$, then there is at most one locally compact group topology on $G$ which is compatible with the duality between $G$ and $H$.

COROLlaRY 4. Let $\left(G, \rho_{1}\right)$ and $\left(G, \rho_{2}\right)$ be locally compact abelian groups with character groups $H_{1}$ and $H_{2}$ respectively. Suppose that $H_{1} \subseteq H_{2}$. Then $\rho_{1} \subseteq \rho_{2}$.

Proof. The topologies $\rho_{1}$ and $\rho_{2}$ are $\pi\left(G, H_{1}\right)$ and $\pi\left(G, H_{2}\right)$ respectively. Clearly $w\left(G, H_{1}\right) \subseteq w\left(G, H_{2}\right)$ so that we have the required inequality $\pi\left(G, H_{1}\right) \subseteq \pi\left(G, H_{2}\right)$.

We remark that this last result is also valid if $\rho_{2}$ is assumed to be a metrisable topology. Corollaries 3 and 4 are certainly not new (see [1], [2], [6] and [7]). However, Corollary 2 would appear to provide the simplest characterisation of this unique topology. In view of Corollary 2 , it is clear that there is a locally compact (group) topology compatible with the duality between $G$ and $H$ if and only if $\pi(G, H)$ is such a topology. We are led to the following theorem.

THEOREM 2. If $(G, H)$ are abelian groups in duality, then there exists a locally compact group topology on $G$ compatible with this duality if and only if

(i) there exists a non-empty, $w(G, H)$-relatively-compact subset $U$ of $G$ for which $K \backslash U$ is $w(G, H)$-compact whenever $K$ is $w(G, H)$-compact, and

(ii) if $\chi$ is a character on $G$ whose restriction to $K$ is $w(G, H)$-continuous for every $w(G, H)$ compact subset $K$ of $G$, then $\chi$ is in $H$.

Proof. If such a topology exists, then it must be $\pi(G, H)$. Then condition (i) just says that there is a compact $\pi(G, H)$-neighbourhood in $G$. On the other hand, condition (ii) tells us that the $\pi(G, H)$-dual of $G$ is contained in $H$. So the conditions are clearly necessary.

Conversely, suppose that (i) and (ii) are satisfied. In view of (i), the topology $\pi(G, H)$ has a relatively compact neighbourhood $U$ of each point of $U$. However, $\pi(G, H)$ is clearly translation invariant, so that it must be a locally compact topology. By Lemma 1 , it is also a group topology. In view of condition (ii), the $\pi(G, H)$-dual of $G$ is contained in, and hence equal to, $H$. 
We remark that a theorem similar to Theorem 2 could be stated, in which the topology $c(G, H)$ plays the role played in the above by $\pi(G, H)$. However, the corresponding versions of conditions (i) and (ii) would become much more complex, involving both the topologies $w(G, H)$ and $w(H, G)$.

Although conditions (i) and (ii) of Theorem 2 are both necessary, it is possible that one of them is automatically satisfied, or that one of them implies the other. We shall give below an example in which condition (ii) is satisfied, although condition (i) is not. Unfortunately, we are unable to give any example in which condition (ii) is not satisfied. However, we remark that this latter condition is to some extent similar to the Banach-Grothendieck condition for completeness in a locally convex topological vector space. Thus it is possible that an example in which $H$ is in some sense " incomplete" can be found, for which (ii) is unsatisfied. The next proposition, which we need for our example, shows how some of the most obvious examples fail to provide a suitable counter-example.

Proposition 1. If $H$ is countable, then the topology $w(G, H)$ is metrisable and

$$
w(G, H)=\pi(G, H) \text {. }
$$

Hence, in this case, condition (ii) of Theorem 2 can be omitted.

Proof. The fact that $w(G, H)$ is metrisable follows by a standard argument. It now suffices to apply the first corollary to Lemma 2.

We now turn to the promised example. Consider the duality $(Q, R)$, where $Q$ is the set of rationals and $R$ is the set of reals, with $r(q)=\exp (i r q)$. Observe that, for the usual duality between $R$ and itself, $\pi(R, R)$ is the usual topology of the reals. We shall show that $\pi(Q, R)$ is the restriction to $Q$ of this topology. Certainly $\left.\pi(Q, R) \supseteq \pi(R, R)\right|_{Q}$. Now $\pi(Q, R)$ and $\left.\pi(R, R)\right|_{Q}$ have the same compact sets, namely the $w(Q, R)$-compact sets. It follows from Lemma $2(a)$ that the metrisable topology $\left.\pi(R, R)\right|_{Q}$ is stronger than $\pi(Q, R)$. Thus these two topologies are equal. It is clear that the set of $\pi(R, R)$-continuous characters on $Q$ is $R$. Hence, for the duality between $Q$ and $R, R$ is the set of continuous characters, so that condition (ii) is satisfied. However $Q$ is not locally compact for $\pi(Q, R)=\left.\pi(R, R)\right|_{Q}$, so that condition (i) is not satisfied. Hence there is no locally compact topology on $Q$ such that the dual group is $R$. By applying the Pontrjagin duality theorem, we see that there is no locally compact topology on $R$ such that the dual is $Q$. In view of Proposition 1 it is clear that again condition (ii) is satisfied but condition (i) is not.

Proposition 2. Let $G$ be a locally compact group, satisfying the second axiom of countability, and let $H$ be its dual group. Then there is no other locally compact Hausdorff group topology on $G$ for which the dual group is contained in $H$.

Proof. Suppose on the contrary that $(G, \pi)$ and $(G, \zeta)$ are locally compact groups, and that the dual of the former contains that of the latter. Suppose also that $(G, \pi)$ satisfies the second axiom of countability. Then we have $\pi \supseteq \zeta$, so that the identity mapping from $(G, \pi)$ to $(G, \zeta)$ is continuous. A suitable version of the closed-graph theorem [5, Corollary 8, page 99] tells us that this mapping is a homeomorphism, so that $\pi=\zeta$. 
In [7] (see also [8]) Ross has posed the question: If $\left(G, \zeta_{1}\right)$ and $\left(G, \zeta_{2}\right)$ are locally compact topological groups, with the same closed subgroups, are they homeomorphic? Our final two results will shed a little light on this problem.

Proposition 3. Let $G$ be a connected locally compact group, with dual group $H$. Then there does not exist a locally compact Hausdorff group topology on $G$ which has the same closed subgroups and has a dual group contained in $H$.

Proof. Since $G$ is connected, $G$ is of the form $R^{n} \times C$, where $R^{n}$ has the usual topology and $C$ is a compact subgroup of $G$. Let $\zeta$ be a topology having the same closed subgroups as $G$, for which the dual group is contained in $H$. Then $R^{n}$ is closed in $G$ for $\zeta$. The two topologies must coincide on $C$. In view of Proposition 2, they also coincide on $R^{n}$. Since the original topology of $G$ is the product topology, the two topologies in fact coincide.

COROLlaRY. Let $G$ be a locally compact group, with dual group $H$. Then there does not exist a locally compact Hausdorff group topology on $G$ which has the same closed subgroups and has dual group contained in $H$.

Proof. First observe that two topologies with the same closed subgroups also have the same open subgroups (see [8, Theorem 1.1]). We may select, in a locally compact topological group, an open subgroup which is of the form $R^{n} \times C$, where $C$ is a compact subgroup of the group. Applying the same arguments as in the proof of Proposition 3, we see that any other locally compact group topology whose dual group is contained in $H$ must agree on that open subgroup, and hence on the whole of $G$.

One might hope to extend this proposition, and the corollary, to show that we cannot have two topologies on a group (both locally compact group topologies) which have the same closed subgroups, and for which the intersection of their dual groups separates points of the group. Unfortunately, it would seem to be necessary to assume that the intersection of the two topologies (see for example [1]) also has the same closed subgroups.

\section{REFERENCES}

1. N. Th. Varopoulos, Studies in harmonic analysis, Proc. Camb. Philos. Soc. 60 (1964), 465-516.

2. I. Glicksberg, Uniform boundedness for groups, Canad. J. Math. 14 (1962), 269-276.

3. Y. Komura, Some examples on linear topological spaces, Math. Ann. 153 (1964), 150-162.

4. T. Husain, Semi-topological groups and linear spaces, Math. Ann. 160 (1965), 146-160.

5. T. Husain, Introduction to topological groups (Philadelphia and London, 1966). $55-64$.

6. E. Hewitt, A remark on characters of locally compact abelian groups, Fund. Math. 53 (1964), 243.

7. K. A. Ross, Closed subgroups of locally compact abelian groups, Fund. Math. 56 (1965), 241$225-235$.

8. N. W. Rickert, Locally compact topologies for groups, Trans. Amer. Math. Soc. 126 (1967),

University COllege of SWANSEa 